\title{
Pulse Train Controlled PCCM Buck-Boost Converter
}

\author{
Ming Qin ${ }^{a}$, Fangfang $\mathrm{Li}^{\mathrm{b}}$ \\ School of Electrical Engineering, Zhengzhou University, Zhengzhou 450001, China \\ aqinming@zzu.edu.cn, b13603714813@163.com
}

Keywords: pulse train (PT) control, pseudo continuous conduction mode (PCCM), Buck-Boost converter, control scheme.

\begin{abstract}
In order to reduce the impact of the decrease of inductor current during the freewheel switch on the pulse of next cycle, this paper puts forward a novel control scheme of pulse train (PT) according to the working character of the pseudo continuous conduction mode (PCCM) Buck-Boost converter. The novel scheme contains two pseudo continuous variables and makes the controller produce different current-controlled pulses depending on different-energy voltage-controlled pulses. The working process of the PCCM Buck-Boost converter under this new control scheme is analyzed in detail and the ratio of high-energy and low-energy voltage-controlled pulses is present. Compared with traditional control scheme with one pseudo continuous variable, the performance of the new converter is improved significantly. Simulation and experimental results verify the feasibility of the new control scheme and the correctness of the theoretical analysis.
\end{abstract}

\section{Introduction}

With the wide application of switching power supply, the requirement of its performance is increasing [1]. The control technology of switching power supply is the main factor that affects its performance. The pulse width modulation (PWM) technique based on linear control theory has gained increasing attention in recent years because of its poor transient characteristics and robustness and difficult design of compensation network [2]. People have been trying to use better control technology to improve the performance of switching power supply to meet the requirements of electronic devices [3, 4].

Pulse train (PT) control technology proposed in recent years is a new nonlinear control technology of switching power supply [5]. It has been widely studied because of its simple control circuit and good transient characteristics and robustness. PT control technique preset high-energy and low-energy voltage-controlled pulses which have the same frequency and different duty ratio. Select suitable pulse as the effective control pulse through the relationship between the output voltage at the initial time of switching cycle and the reference voltage. Some high-energy and low-energy voltage-controlled pulses form a pulse train cycle and the controller adjusts the output voltage by adjusting the combination of the two pulses in pulse train cycle.

The switching converter operating at a constant frequency can work in discontinuous conduction mode (DCM) or continuous conduction mode (CCM). At present, the research of PT control technology is mostly based on the DCM converter, whose ability of carrying capacity is poor. Pseudo continuous conduction mode (PCCM) is a special operating mode different from DCM and CCM [6]. At any time the inductor current is greater than zero, and the inductor current is kept constant rather than zero before the end of the switching cycle.

Considering the equivalent series resistance of inductor and capacitor, this paper puts forward a new pulse train control strategy, which makes the Buck-Boost converter work in PCCM mode. The control process and high-energy and low-energy voltage-controlled pulses are analyzed. Finally, the feasibility of the method and the correctness of the theoretical analysis are verified by simulation and experiment. 


\section{PT Controlled PCCM Buck-Boost Converter}

Fig. 1 shows the circuit structure diagram of PT controlled PCCM Buck-Boost converter. The diode $D_{2}$ and the switch $S_{2}$ are in parallel with the inductor $L$, which could provide a continued flow path for current. Converter can work in PCCM through controlling the on-off of the two switches. There are three parts in each switching cycle of a PCCM Buck-Boost converter. When the switch $S_{1}$ is on and $S_{2}$ is off, the inductor current $i_{L}$ and the output voltage $V_{o}$ ramp up. When $S_{1}$ and $S_{2}$ are off, $i_{L}$ ramps up and. When $S_{1}$ is off and $S_{2}$ is on, $i_{L}$ stays the same and $V_{o}$ ramps down.

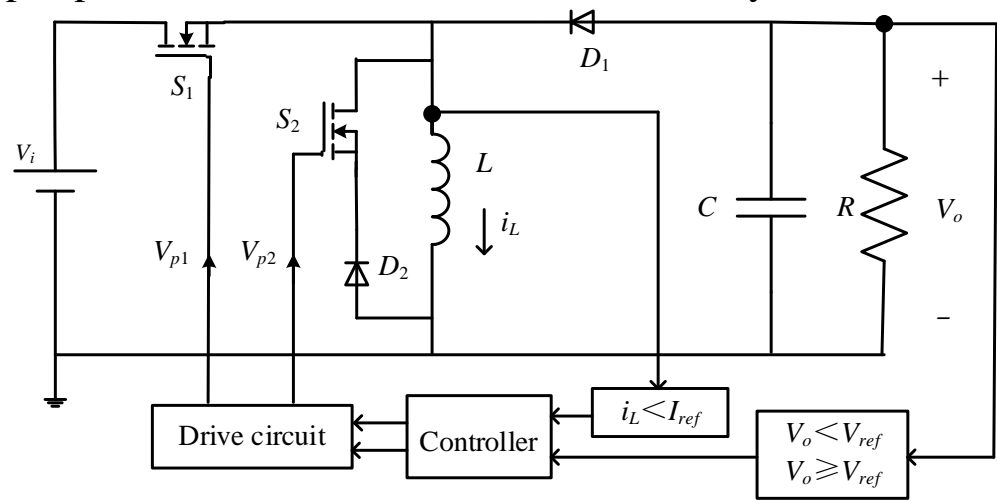

Fig. 1 The circuit structure diagram of PT controlled PCCM Buck-Boost converter

Actually, the inductor current will be linearly decreased due to the loss of the inductance and the switch. Considering this losses, this paper proposes a new control strategy of PT to make the Buck-Boost converter work in PCCM, which has two pseudo continuous variables. The controller is composed of a pulse train controller and a pseudo continuous controller. In the beginning of each switching cycle, $V_{o}$ is compared with the reference voltage $V_{\text {ref. }}$. When $V_{o}$ is lower than $V_{\text {ref }}$, pulse train controller generates high-energy voltage-controlled pulse $P_{H 1}$ to control $S_{1}$. When $i_{L}$ drops to the reference current $I_{r e f}$, the pseudo continuous controller generates the current-controlled pulse $P_{H 2}$ to make $S_{2}$ turn on, then $i_{L}$ freewheels through $S_{2}$ and $D_{2}$ until the next switching cycle begins. When $V_{o}$ is higher than $V_{\text {ref }}$, the pulse train controller generates low-energy voltage-controlled pulse $P_{L 1}$ to control $S_{1}$. When this cycle has worked for a certain period of time $t_{r e f}$, the pseudo continuous controller generates the current-controlled pulse $P_{L 2}$ to make $S_{2}$ turn on, which is different from the period of $P_{H 1}$, then $i_{L}$ freewheels through $S_{2}$ and $D_{2}$ until the next switching cycle begins. Fig. 2 shows the control flow chart of this novel control scheme.

There is only one pseudo continuous variable $I_{r e f}$ in traditional PCCM converter. Whether the pulse train controller produces $P_{H 1}$ or $P_{L 1}$, the pseudo continuous controller will produce corresponding current-controlled pulse to control the conduction of $S_{2}$ when $i_{L}$ drops to $I_{r e f}$. But in practice, for the time of freewheel switch of $P_{L 1}$ is long, the inductor current drops a lot, which will seriously affect the next cycle. For the time of freewheel switch of $P_{H 1}$ is short, the inductor current drops can be ignored. The inductor current waveforms of these two cases are shown in Fig. 3.

The novel control strategy adds one time control volume on the basis of a reference current, which can't affect next cycle when the pulse train controller produces $P_{L 1}$ and the control performance can be improved significantly. Fig. 4 shows the main waveforms of this PT controlled Buck-Boost PCCM converter with two pseudo continuous variables. 


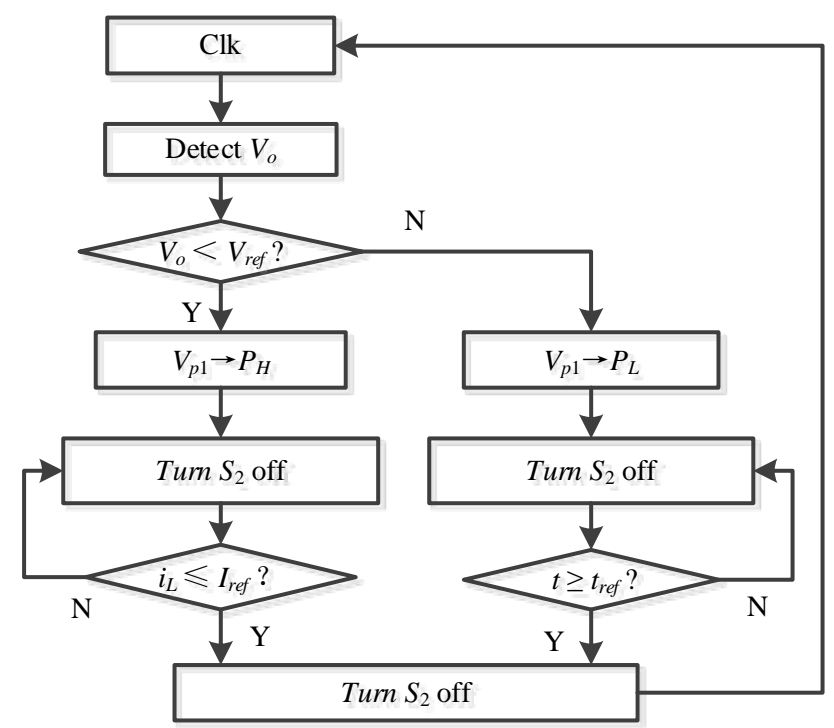

Fig. 2 The control flow chart

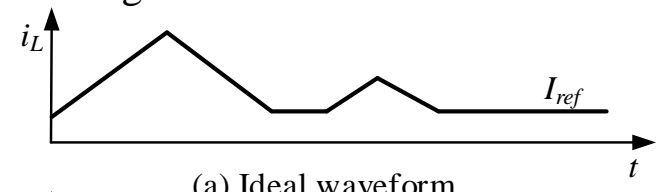

(a) Ideal waveform

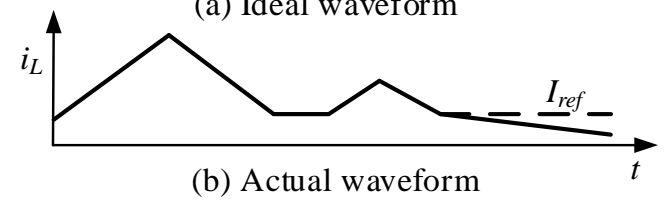

Fig. 3 Inductor current waveforms with one pseudo continuous variable

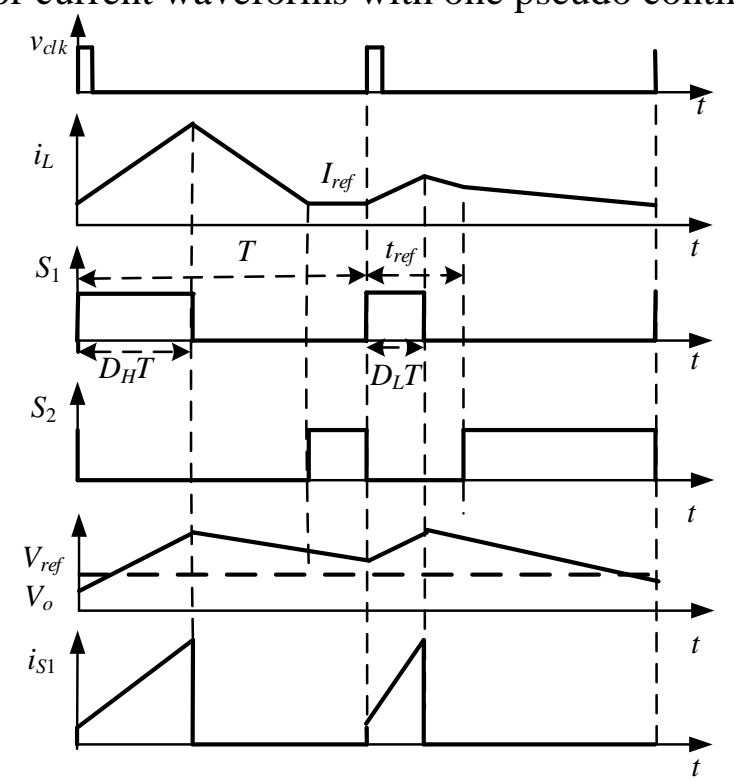

Fig. 4 Main waveforms with two pseudo continuous variables

\section{High-energy and Low-energy Voltage-controlled Pulses}

For the PT controlled PCCM Buck-Boost converter, during the switch cycle of $P_{H 1}$, the conduction time of $S_{1}$ is $D_{H} T$, the waveform of which is shown in Fig. 4. The mean value of the current flowing through the $\mathrm{S} 1$ during the switching period is

$$
I_{S 1 H}=I_{\text {ref }} D_{H}+\frac{T V_{\mathrm{i}} D_{H}^{2}}{2 L},
$$

where $L$ is the inductance of converter.

The input terminal current of the converter is the current that flows through $S_{1}$, so the energy obtained from the input during the switching cycle $P_{H 1}$ is 


$$
E_{i H}=V_{i} I_{r e f} D_{H} T+\frac{\left(V_{i} D_{H} T\right)^{2}}{2 L} \text {. }
$$

Similarly, the energy obtained from the input during the switching cycle $P L_{1}$ is

$$
E_{i L}=V_{i} I_{r e f} D_{L} T+\frac{\left(V_{i} D_{L} T\right)^{2}}{2 L} \text {. }
$$

Assuming the pulse train cycle is composed of $\mu_{H}$ high-energy voltage-controlled pulses and $\mu_{L}$ low-energy voltage-controlled pulses when the converter is stable. The total energy of the converter obtained from the input of the pulse train cycle is

$$
\sum E_{i}=\mu_{H} E_{i H}+\mu_{L} E_{\mathrm{i} L} \text {. }
$$

Assuming the load power of the converter is $P$ and the conversion efficiency of energy is $\eta$. There is the following relationship

$$
\eta \sum E_{i}=P \cdot\left(\mu_{H}+\mu_{L}\right) T \text {. }
$$

By equation (4) and equation (5), we obtain

$$
P=\frac{1}{2 L\left(\mu_{H}+\mu_{L}\right)}\left[2 L \eta V_{\mathrm{i}} I_{r e f}\left(\mu_{H} D_{H}+\mu_{L} D_{L}\right)+V_{\mathrm{i}}^{2} T \eta\left(\mu_{H} D_{H}^{2}+\mu_{L} D_{L}^{2}\right)\right] \text {. }
$$

The equation (6) shows the main circuit parameters and the control parameters of the converter and the quantitative relation of high-energy and low-energy voltage-controlled pulses in the pulse train cycle, which can be used as reference in the design of control system.

According to the analysis method of PCCM converter, we can obtain the load power of pulse train cycle in DCM, that is

$$
P=\frac{V_{i}^{2} T \eta\left(\mu_{H} D_{H}^{2}+\mu_{L} D_{L}^{2}\right)}{2 L\left(\mu_{H}+\mu_{L}\right)} .
$$

By comparing equation (6) and equation (7), we know that the load power of the PCCM is higher than that of the DCM under the same parameters of converter.

We can get the quantity proportion between $P_{H 1}$ and $P_{L 1}$ of pulse train cycle of the PCCM. The equation is

$$
\frac{\mu_{H}}{\mu_{L}}=\frac{2 L P-2 L \eta V_{\mathrm{i}} I_{r e f} D_{L}-\eta V_{i}^{2} D_{L}^{2} T}{2 L \eta V_{i} I_{r e f} D_{H}+\eta V_{i}^{2} D_{H}^{2} T-2 L P} .
$$

Similarly, we can get the quantity proportion between $P_{H 1}$ and $P_{L 1}$ of PT controlled DCM converter from equation (7). The equation is

$$
\frac{\mu_{H}}{\mu_{L}}=\frac{2 L P-\eta V_{i}^{2} D_{L}^{2} T}{\eta V_{i}^{2} D_{H}^{2} T-2 L P} .
$$

From equation (8) and (9), we can draw the following conclusions. If the input voltage is constant while the load power is increased, the number of $P_{H 1}$ in pulse train controller would relatively increase. If the load power is constant and the input voltage rises, pulse train controller would generate more $P_{L 1}$.

We can draw the relation curve between $\mu_{H} / \mu_{L}$ and $P$ of PT controlled PCCM Buck-Boost converter and PT controlled DCM Buck-Boost converter from equation (8) and (9), which is shown in Fig. 5. Where, $V_{i}=10 \mathrm{~V}, V_{o}=9 \mathrm{~V}, P=1.8 \mathrm{~W}, T=50 \mu \mathrm{S}, L=100 \mu \mathrm{H}, C=470 \mu \mathrm{F}, D_{H}=0.12, D_{L}=0.4$ and $I_{\text {ref }}=0.5 \mathrm{~A}$.

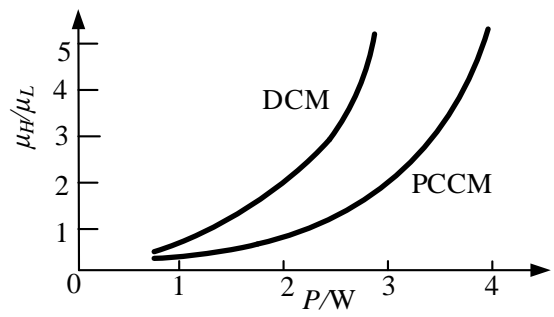

Fig. 5 Relation curve between $\mu_{H} / \mu_{L}$ and $P$ 
Fig. 5 shows that $\mu_{H} / \mu_{L}$ increases with the increase of $P$. When $P$ is increased to a certain value, $\mu_{H} / \mu_{L}$ increases rapidly and the converter can't work normally. The range of load power of PCCM is greater than DCM. It can be concluded that, PCCM converter improves the load capacity compared with DCM converter.

\section{Simulation Research}

In order to prove the correctness of the theoretical analysis, the PT controlled PCCM Buck-Boost converter with two pseudo continuous variables is simulated, whose circuit parameters and control parameters are the same with the last section.

Fig. 6 shows the simulation waveforms of voltage-control pulses of the PCCM converter under the same input voltage and different load power. When $P$ is $1.8 \mathrm{~W}$, the pulse train cycle is $P_{H 1}-P_{L 1}-P_{L 1}-P_{H 1}-P_{L 1}, \mu_{H} / \mu_{L}=2 / 3$. When $P$ is $1.2 \mathrm{~W}$, the pulse train cycle is $P_{H 1}-P_{L 1}-\mathrm{P}_{L 1}-P_{L 1}$, $\mu_{H} / \mu_{L}=1 / 3$. When $P$ is $1.2 \mathrm{~W}$, the pulse train cycle is $P_{H 1}-P_{H 1}-P_{H 1}-P_{L 1}, \mu_{H} / \mu_{L}=3$.

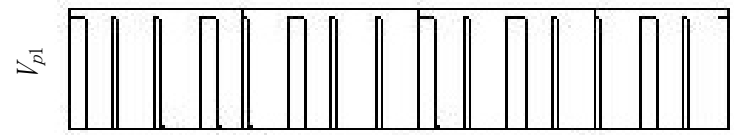

(a) $P$ is $1.8 \mathrm{~W}$

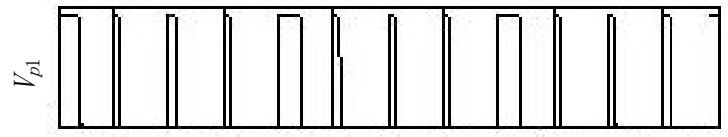

(b) $P$ is $1.4 \mathrm{~W}$

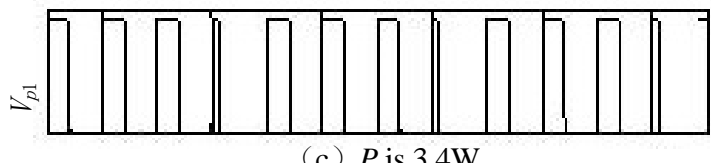

Fig. 6 Simulation waveforms

The simulation results show that the pulse train controller generates more low-energy voltage-controlled pulses when the load power is light and the pulse train controller generates more high-energy voltage-controlled pulses when the load power is heavy. In addition, PT controlled Buck-Boost DCM converter can't work properly when $P$ is $3.4 \mathrm{~W}$, which indicates that the PCCM converter can improve the load carrying capacity compared to DCM converter. The simulation results verify the correctness of the theoretical analysis.

\section{Experimental Verification}

In order to verify the feasibility of the new control strategy and the correctness of the theoretical analysis and simulation research, this paper build the experiment platform to test the PT controlled PCCM Buck-Boost converter with two pseudo continuous variables, whose experimental parameters are the same with simulation parameters. Switches use IRF3205. Diodes use SR560. Comparators use LM393. Photoelectric couplers use 6N137. Controller uses digital controller FPGA, whose model is EP4CE15F17C8. Drivers use A3120.

Fig. 7 shows the experimental waveforms of voltage-controlled pulses and inductor current when the load power of the PCCM converter is $1.8 \mathrm{~W}, 1.2 \mathrm{~W}$ and $3.4 \mathrm{~W}$, respectively. The pulse train cycle is $P_{H 1}-P_{L 1}-P_{L 1}-P_{H 1}-P_{L 1}, P_{H 1}-P_{L 1}-P_{L 1}-P_{L 1}$ and $P_{H 1}-P_{H 1}-P_{H 1}-P_{L 1}$, respectively. 


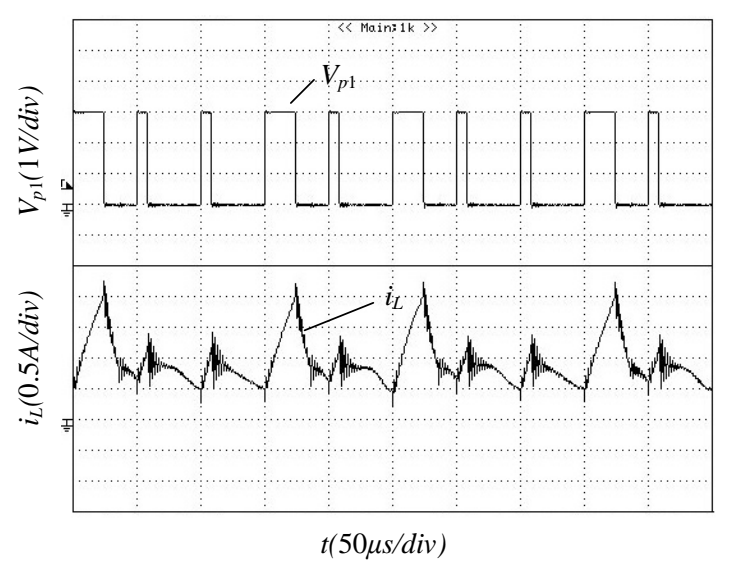

(a) $P$ is $1.8 \mathrm{~W}$

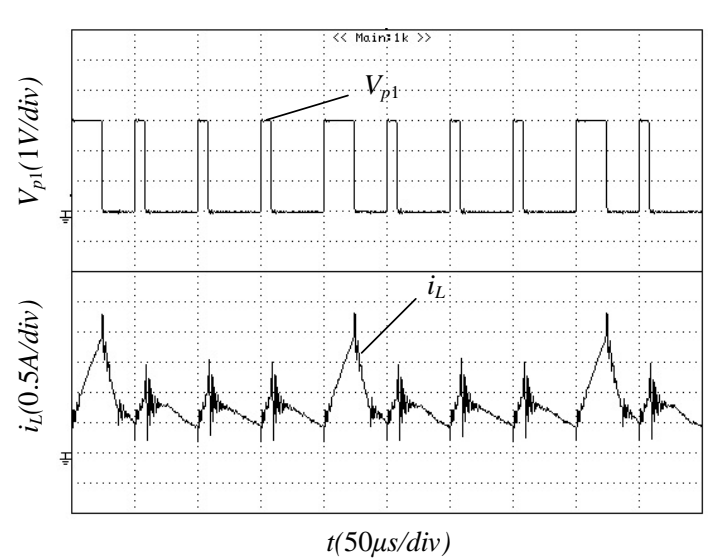

(b) $P$ is $1.2 \mathrm{~W}$

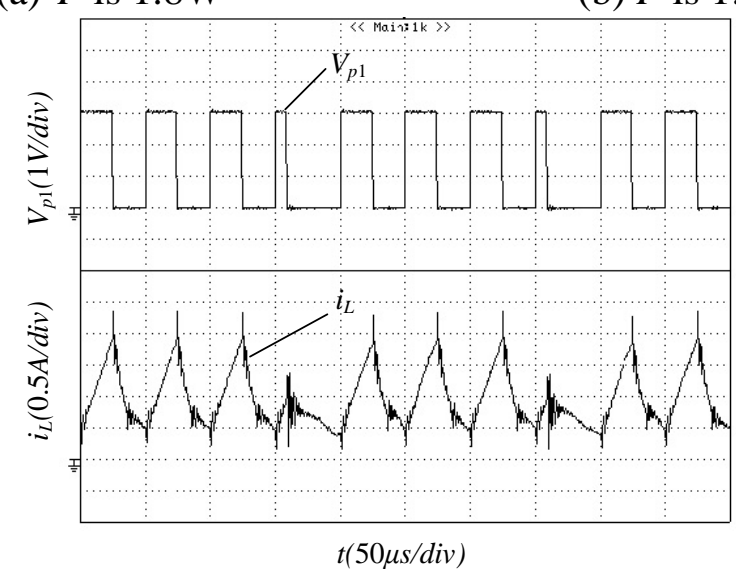

(c) $P$ is $3.4 \mathrm{~W}$

Fig. 7 experimental waveforms

The experimental results are in agreement with the theoretical analysis and simulation results. The high-energy voltage-controlled pulse generated by the controller is relatively increased with the increase of load power and decline with the decrease of load power. The experimental results verify the feasibility of the new control scheme and the correctness of theoretical analysis and simulation research.

\section{Summary}

Traditional PT controlled PCCM converter has only one pseudo continuous variable. Based on this, this paper proposes a novel PT controlled strategy with two pseudo continuous variables. The controller generates corresponding current-controlled pulses according to the voltage-controlled pulses, which makes the performance of the converter improved significantly. The working process of the converter is analyzed in detail. The operation state and pulse combination of PT controlled Buck-Boost PCCM converter is analyzed through the simulation and experimental research. The feasibility of the new control method and the correctness of the theoretical analysis are verified. At the same time, it is proved that the load carrying capacity of PT controlled PCCM converter is significantly improved compared with the PT controlled DCM converter.

\section{Acknowledgments}

This research is financially supported by the National Natural Science Foundation (51207142) of China and China Postdoctoral Science Foundation (2012M521408). 


\section{References}

[1] C Chang. Mixed voltage/current mode control of PWM synchronous buck converter. Power Electronics and Motion Control Conference, 2004. IPEMC 2004. The 4th International. Xi'an, China, 2004, p. 1136-1139.

[2] F C Lee, R A Carte. Investigations of stability and dynamic performances of switching regulators employing current-injected control. Power Electronics Specialists Conference. Boulder, Colorado, USA, 1981, p. 3-16.

[3] Z Wu, H Li, P Zuo, et al. Cascade control of DC/DC boost converters. Proceedings of the CSEE. Vol. 22 (2002) No. 1, p. 110-115.

[4] Y Ni, J P Xu. Design of a novel discrete global sliding mode controlled Buck converter. Electric Machines and Control. Vol. 13 (2009) No. 1, p. 112-116.

[5] M Qin, J P Xu, Q B Mou. Control laws and characters of pulse train controlled Buck converters. Journal of Southwest Jiaotong University. Vol. 44 (2009) No. 5, p. 660-666.

[6] F Zhang, J P Xu, P Yang, et a1. Two-switch pseudo continuous conduction mode Buck-Boost power factor correction converter. Proceedings of the CSEE. Vol. 32 (2012) No. 9, p.56-64. 